uptake and long-term satisfaction. Int. J. Cancer 89, 44-50 (2000).

6. University of California at San Francisco. Lynch Syndrome Kintalk@UCSF [online], http:// kintalk.org (2013).

7. Lynch Syndrome International. Lynch Syndrome International [online], www.lynchcancers.com (2013).

8. GINA Genetic Information Nondiscrimination Act [online], www.ginahelp.org (2013).

9. Genetic Alliance, the Genetics and Public Policy Center at the Johns Hopkins
University, and the National Coalition for Health Professional Education in Genetics through funding by The Pew Charitable Trusts. National Human Genome Research Institute. The Genetic Information Nondiscrimination Act (GINA) of 2008 [online], http://www.genome.gov/ 24519851 (2012).

10. Lapointe, J. et al. Life events may contribute to family communication about cancer risk following BRCA1/2 testing. J. Genet. Couns. 22, 249-257 (2013).

\title{
VIRAL HEPATITIS
}

\section{Cell-culture-derived HCV -a promising vaccine antigen}

Judith M. Gottwein and Jens Bukh

\begin{abstract}
With millions of humans infected yearly with HCV, leading to cirrhosis and cancer, a vaccine is urgently needed. Cultured virus particles constitute the antigen in most antiviral vaccines. A study in mice demonstrated induction of neutralizing antibodies by immunization with cell-culturederived HCV, providing new possibilities for vaccine development.
\end{abstract}

Gottwein, J. M. \& Bukh, J. Nat. Rev. Gastroenterol. Hepatol. 10, 508-509 (2013); published online 30 July 2013; doi:10.1038/nrgastro.2013.136

Approximately 3-4 million new HCV infections occur yearly, with $>150$ million chronic infections worldwide. ${ }^{1,2}$ Efficient therapeutics that target specific HCV proteins, defined steps of the HCV life cycle or essential host factors are under development. ${ }^{3}$ However, owing to the asymptomatic nature of most infections, economic constraints, as well as drug adverse effects and contraindications, only a minority of $\mathrm{HCV}$-infected individuals will be diagnosed and treated. A vaccine is urgently needed to prevent $\mathrm{HCV}$ infection globally. Akazawa et al. ${ }^{4}$ have now provided proof-of-concept for immunogenicity of cell-culture-derived HCV particles (HCVcc). HCVcc of genotype 2a were concentrated, purified, UV-inactivated and formulated with an experimental oil-water adjuvant. Four consecutive immunizations of $\mathrm{BALB} / \mathrm{c}$ mice elicited antibodies with neutralizing capacity against HCV genotypes $1 \mathrm{a}$, $1 \mathrm{~b}$ and $2 \mathrm{a}$ in vitro, and with an apparent protective effect against genotype 2 a HCVcc infection in a human liver chimeric mouse model. Thus, HCVcc seems to be a promising antigen for a whole-virus, inactivated HCV vaccine.

Protective immunity against $\mathrm{HCV}$ exists- $25 \%$ of acutely infected individuals clear $\mathrm{HCV}$ - and, in some cases, protection from reinfection was observed. ${ }^{1,2,5}$ From early studies in humans and chimpanzees (considered the only true animal model for studies of $\mathrm{HCV}$ infection ${ }^{6}$ ), activated $\mathrm{HCV}$ specific $\mathrm{CD}^{+}$and $\mathrm{CD} 8^{+} \mathrm{T}$ cells are required for control of HCV infection. The development of culture systems, specifically HCV pseudo-particles (HCVpp) and $\mathrm{HCVcc}{ }^{3}$ enabled in vitro studies of $\mathrm{HCV}$ neutralizing antibodies; evidence was subsequently obtained that neutralizing antibodies are of importance for HCV clearance. ${ }^{1,2,5}$ Indeed, most antiviral vaccines use inactivated and/or attenuated whole viral particles as antigen and depend, at least in part, on the induction of neutralizing antibodies. HCV vaccine research has focused on recombinant protein-based, peptide-based, DNAbased, vector-based, or virus-like-particle vaccine candidates, with the most advanced candidates in phase II clinical trials. ${ }^{1,2}$

Vaccine candidates containing adjuvantformulated HCV envelope glycoproteins (E1 and E2), or virus-like-particles containing $\mathrm{HCV}$ envelope proteins, most closely resemble a whole-HCV particle vaccine. Immunization with virus-like-particles induced humoral and cellular immune responses in several animal models; however, no HCV-specific antibodies were induced in chimpanzees. ${ }^{1,2}$ Although immunizations with heterodimeric E1-E2 induced strong humoral immune responses in chimpanzees and humans (phase I trial), neutralizing antibody responses were detected in $<50 \%$ of immunized humans. ${ }^{1,2}$
Of note, production of these heterodimers seems to be technically difficult. DNA-based or vector-based vaccines primarily induce cellular immunity, which might limit their use as a prophylactic vaccine. The efficacy of viral-vector-based vaccines might also be compromised by pre-existing antibodies neutralizing the viral vector. ${ }^{1,2}$ These limitations of prophylactic $\mathrm{HCV}$ vaccine candidates might restrict their use, and their protective effect in humans is yet to be established.

In 2005, the first cell-culture system to produce infectious HCV was developed, which enabled the same research group to study HCVcc immunogenicity in mice (Figure 1). ${ }^{7}$ Immunization with $\mathrm{HCVcc}$ of genotype $2 \mathrm{a}$ with an adjuvant elicited anti-E1 and anti-E2 antibodies, as well as HCV neutralizing antibodies. ${ }^{4}$ Serum from immunized mice had 50\% neutralization titres of $\sim 1: 100$ against HCVpp of genotype $1 \mathrm{a}, 1 \mathrm{~b}$ and $2 \mathrm{a}$ and $\sim 1: 500$ against HCVcc of genotype $1 b$ and $2 a$; in the latter experiment, however, pre-immunization control sera were not included. The serum 50\% HCVpp neutralization titres were comparable to those found in chimpanzees and humans after immunization with the E1-E2 vaccine candidate. ${ }^{2}$ Polyclonal IgG purified from immunized mice neutralized HCVpp of genotype $1 \mathrm{~b}$ and $2 \mathrm{a}$ and HCVcc of genotype $2 \mathrm{a}$, with $\mathrm{EC}_{50}$ values in the upper range of those observed for human monoclonal antibodies with therapeutic potential. ${ }^{4,5}$ Of note, pre-absorption of anti-E1 and anti-E2 antibodies in purified polyclonal IgG with recombinant $\mathrm{E} 1$ and $\mathrm{E} 2$ resulted only in a $\sim$ threefold increase in $\mathrm{EC}_{50}{ }^{4}$ Neutralizing antibodies, therefore, seem to target not only the HCV envelope proteins but also other components of the vaccine preparation. Although whole-virus vaccines mainly elicit humoral immune responses, any T-cell responses triggered by HCVcc-based vaccines should also be studied (Figure 1).

Experimental use of chimpanzees has been restricted, and it is unclear if this animal model will be available for HCV immunization and challenge studies in the future (Figure 1). ${ }^{6}$ The only small animal model to support robust HCV infection are SCID-uPA mice, whose auto-degenerating liver can be repopulated with human hepatocytes (Figure 1). ${ }^{6}$ Polyclonal IgG from BALB/c mice immunized with $\mathrm{HCV} c \mathrm{c}$ of genotype $2 \mathrm{a}$ apparently had a protective effect against challenge with HCVcc of genotype $2 \mathrm{a}$ in this mouse model. ${ }^{4}$ When challenged with the minimal infectious 


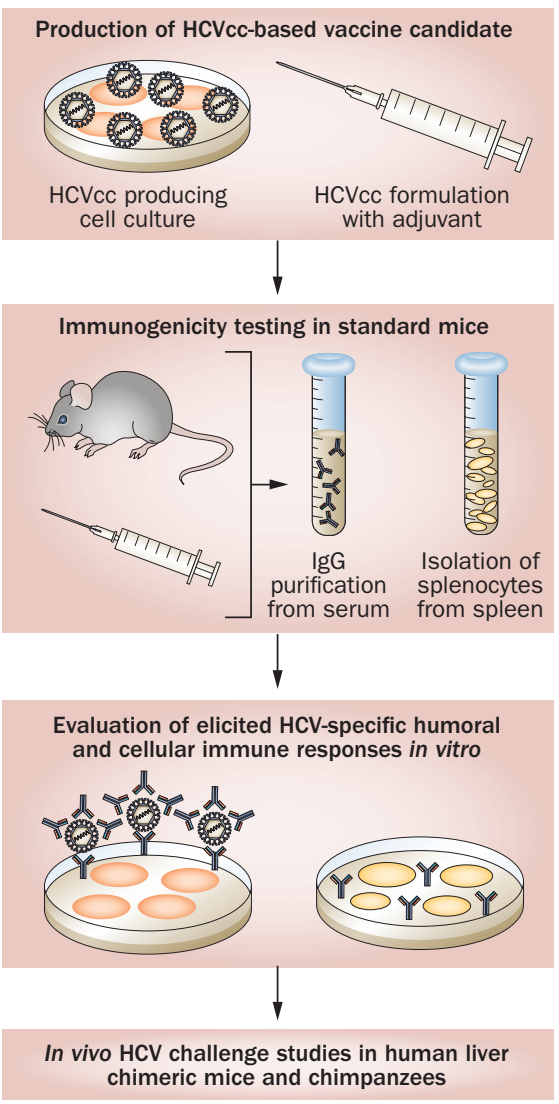

Figure 1 | Development of a whole-virus, inactivated $\mathrm{HCV}$ vaccine candidate.

dose (equivalent to $10^{3} \mathrm{HCV}$ RNA copies), but not with higher doses, all of the IgGloaded mice $(n=6)$ were HCV negative post-challenge, whereas four of the control mice $(n=6)$ became infected. Whether the observed protective effect was conveyed by IgG in serum of challenged mice remains to be determined, as mixing of challenge virus with IgG might lead to in vitro viral neutralization. ${ }^{8}$ The polyclonal IgG dose of $100 \mu \mathrm{g}$ given in this study compares favourably to previous in vivo passive immunization studies, in which polyclonal IgG from a patient with chronic $\mathrm{HCV}$ achieved protection in human liver chimeric mice at a dose of $0.2-1 \mathrm{mg}$ per g body weight. ${ }^{8}$

It will be interesting to investigate if $\mathrm{HCVcc}$-vaccine-induced IgG protects against challenge with native HCV particles derived from in vivo infections, which might differ from HCVcc. Indeed, $\mathrm{HCV} c \mathrm{c}$ are believed to resemble the native $\mathrm{HCV}$ particle. ${ }^{2}$ Thus, it was encouraging that, when tested head-tohead with recombinant E1 and E2 protein using the same adjuvant, HCVcc proved to be a more potent antigen regarding induction of neutralizing antibodies. ${ }^{4}$ However, the recombinant E1 and E2 used by Akazawa et al. ${ }^{4}$ differed from extracted and purified intracellular heterodimeric E1-E2 previously used as vaccine antigen. ${ }^{1,2}$ Different antigens might show optimal efficacy in different adjuvants.

Although HCV serotypes have not been defined, different genotypes and subtypes show differential sensitivity to neutralizing antibodies in vitro. ${ }^{9}$ Single amino acid changes can also alter susceptibility to neutralizing antibodies, facilitating HCV immune escape. ${ }^{5} \mathrm{HCV} c \mathrm{c}$ of genotype $2 \mathrm{a}$ showed resistance to neutralization by patient sera, which could be overcome by human monoclonal antibodies and also by polyclonal IgG from mice immunized with HCVcc of genotype $2 \mathrm{a}^{4,10}$ Whether induced antibodies target conserved neutralizing epitopes is currently unknown. ${ }^{5}$

Given the high regional prevalence of HCV genotypes 3-6, it should be determined if antibodies induced by HCVcc of genotype 2a, capable of neutralizing $\mathrm{HCV}$ genotype 1 and 2, are also able to neutralize these genotypes. The immunogenic potential of HCVcc of other major genotypes should also be tested, ${ }^{9}$ as a multivalent vaccine might be required to efficiently protect against all major HCV genotypes. ${ }^{1}$ Lastly, HCV particles engineered to unshield neutralizing epitopes should be developed and tested. For example, HCV particles without the E2 motif HVR1 (which apparently shields susceptible neutralizing epitopes) showed increased sensitivity to neutralizing antibodies, and so might be useful in vaccine development. ${ }^{5}$

Akazawa et al. ${ }^{4}$ used the experimental oil-water based adjuvant MPL-TDM, which is not licensed for human vaccinations. To develop a whole-virus, inactivated vaccine, adjuvants suitable for humans, able to elicit strong humoral and possibly also cellular immune responses, need to be found. Furthermore, this experimental vaccine was administered intraperitoneally; for human use other routes of immunization need to be explored. As the use of components of animal origin should be limited in human vaccine development, it is of importance to establish cell-culture conditions that enable production of large amounts of $\mathrm{HCVcc}$ without the use of bovine serum. Further optimization of HCVcc purification procedures might also be required before this antigen can be used in humans.

This study provides evidence that immunization of mice with $\mathrm{HCVcc}$ results in induction of neutralizing antibodies, able to protect against HCVcc infection in cell culture and in human liver chimeric mice. These promising findings warrant exploration of this novel
HCV vaccine approach, including immunogenicity studies in mice to determine the effect of different HCVcc antigens and adjuvants and an analysis of elicited neutralizing antibody and T-cell responses (Figure 1). The protective effect of promising vaccine candidates needs to be tested in high incidence populations in clinical trials. Eventually, a whole-viral-particle vaccine might benefit from combination with technologies that primarily elicit T-cell responses, such as viral-vector-based vaccines. ${ }^{1}$

Copenhagen Hepatitis C Program (CO-HEP), Department of Infectious Diseases and Clinical Research Centre, Copenhagen University Hospital, Hvidovre, Kettegaard Alle 30, DK-2650 Hvidovre, Denmark and Department of International Health, Immunology and Micobiology, Faculty of Health and Medical Sciences, University of Copenhagen, Blegdamsvej 3B, DK-2200, Copenhagen N., Denmark (J. M. Gottwein, J. Bukh).

Correspondence to: J. Bukh

jbukh@sund.ku.dk

Competing interests

The authors declare no competing interests.

1. Swadling, L., Klenerman, P. \& Barnes, E. Ever closer to a prophylactic vaccine for HCV. Expert Opin. Biol. Ther. 13, 1109-1124 (2013).

2. Fauvelle, C. et al. Hepatitis $C$ virus vaccines -Progress and perspectives. Microb. Pathog. 58, 66-72 (2013).

3. Bartenschlager, R., Lohmann, V. \& Penin, F. The molecular and structural basis of advanced antiviral therapy for hepatitis $C$ virus infection. Nat. Rev. Microbiol. 11, 482-496 (2013).

4. Akazawa, D. et al. Neutralizing antibodies induced by cell culture-derived hepatitis $C$ virus protect against infection in mice. Gastroenterology http://dx.doi.org/10.1053/ j.gastro.2013.05.007.

5. Wahid, A. \& Dubuisson, J. Virus-neutralizing antibodies to hepatitis $C$ virus. J. Viral Hepat. 20, 369-376 (2013).

6. Bukh, J. Animal models for the study of hepatitis $C$ virus infection and related liver disease. Gastroenterology 142, 1279-1287 (2012).

7. Wakita, T. et al. Production of infectious hepatitis $C$ virus in tissue culture from a cloned viral genome. Nat. Med. 11, 791-796 (2005).

8. Vanwolleghem, T. et al. Polyclonal immunoglobulins from a chronic hepatitis $\mathrm{C}$ virus patient protect human liver-chimeric mice from infection with a homologous hepatitis $C$ virus strain. Hepatology 47, 1846-1855 (2008).

9. Gottwein, J. M. et al. Development and characterization of hepatitis $C$ virus genotype 1-7 cell culture systems: role of CD81 and scavenger receptor class B type I and effect of antiviral drugs. Hepatology 49, 364-377 (2009).

10. Pedersen, J. et al. Neutralization resistance of hepatitis $C$ virus can be overcome by recombinant human monoclonal antibodies. Hepatology http://dx.doi.org/10.1002/ hep. 26524. 